\title{
LIQUID BRIDGE BREAKAGES ABOARD SPACELAB-D1
}

\author{
José MESEGUER, Angel SANZ and Jesús LOPEZ \\ Laboratorio de Aerodinámica, ETSI Aeronáuticos, Universidad Politécnica, 28040 Madrid, Spain
}

Received 19 March 1986; manuscript received in final form 20 July 1986

The study of the stability of long liquid columns under microgravity was the purpose of one of the experiments carried out aboard Spacelab-D1. In this paper a preliminary analysis of this experiment, mainly concerning the different liquid column breakages, is presented, As shown in the paper, the behaviour, both static and dynamic, of long liquid bridges can be accurately predicted by using available theoretical models.

\section{Introduction}

The experiment Floating Liquid Zone (FLIZ), dealing with the behaviour of long liquid bridges under mechanical disturbances in a low gravity environment, was carried out by the german payload specialist Dr. R. Furrer during the Spacelab-D1 Mission in November 1985 [1]. This experiment was performed in the Fluid Physics Module (FPM), one of the multiuser facilities provided by the European Space Agency for experimentation aboard spacecraft [2].

The configuration of the FLIZ experiment consisted in a volume of liquid held by surface tension forces between two equal diameter, coaxial solid disks, as sketched in fig. 1. The disks were made of aluminium, which a $30^{\circ}$ sharp edge to avoid liquid spreading, the disk radius being $R_{0}=$ $0.0175 \mathrm{~m}$. Working liquid was a low viscosity density $\rho=920 \mathrm{~kg} \mathrm{~m}^{-3}$ and surface tension $\sigma=$ $0.02 \mathrm{~N} \mathrm{~m}^{-1}$ ) with tracers (Eccospheres, $0.15 \times$ $10^{-3} \mathrm{~m}$ in diameter and $0.1 \mathrm{~kg} \mathrm{~m}^{-3}$ of concentration) to enhance inner motion visualization.

During the FLIZ experiment, the liquid bridge broke down six times, and these breakages are analyzed in this paper by comparing experimental results with available theoretical ones. As published elsewhere [3-6], concerning liquid bridge breakage, the most suitable result to be experimentally checked is the partial volume (defined as the ratio of the volume of the larger drop appearing after liquid bridge breakage to the whole liquid column volume), because the partial volume depends mainly on variables which can be easily controlled and experimentally measured (volume of the liquid column and the slenderness $\Lambda=$

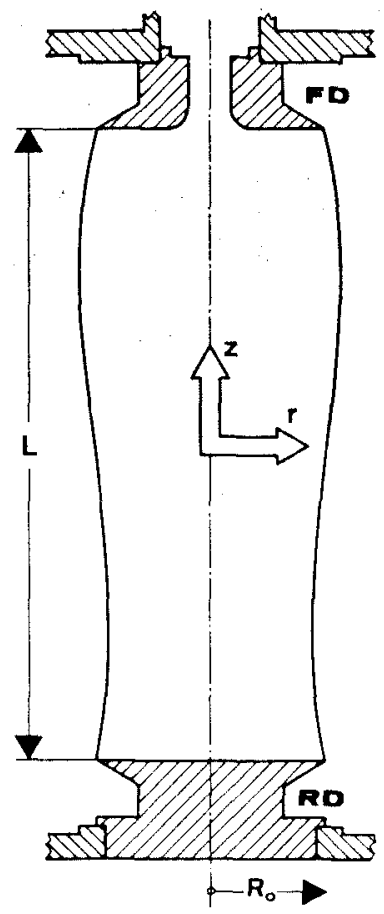

Fig. 1. Sketch of the liquid bridge (FLIZ experiment, SpacelabD1). FD: feeding disk: RD: rear disk. 
$L /\left(2 R_{0}\right)$, where $L$ stands for the distance between the disks). On the other hand, some other aspects of the liquid bridge breakage (i.e. the breaking time) are extremely difficult to check because of the strong dependence of these results on initial conditions, which are too hard to control accu-
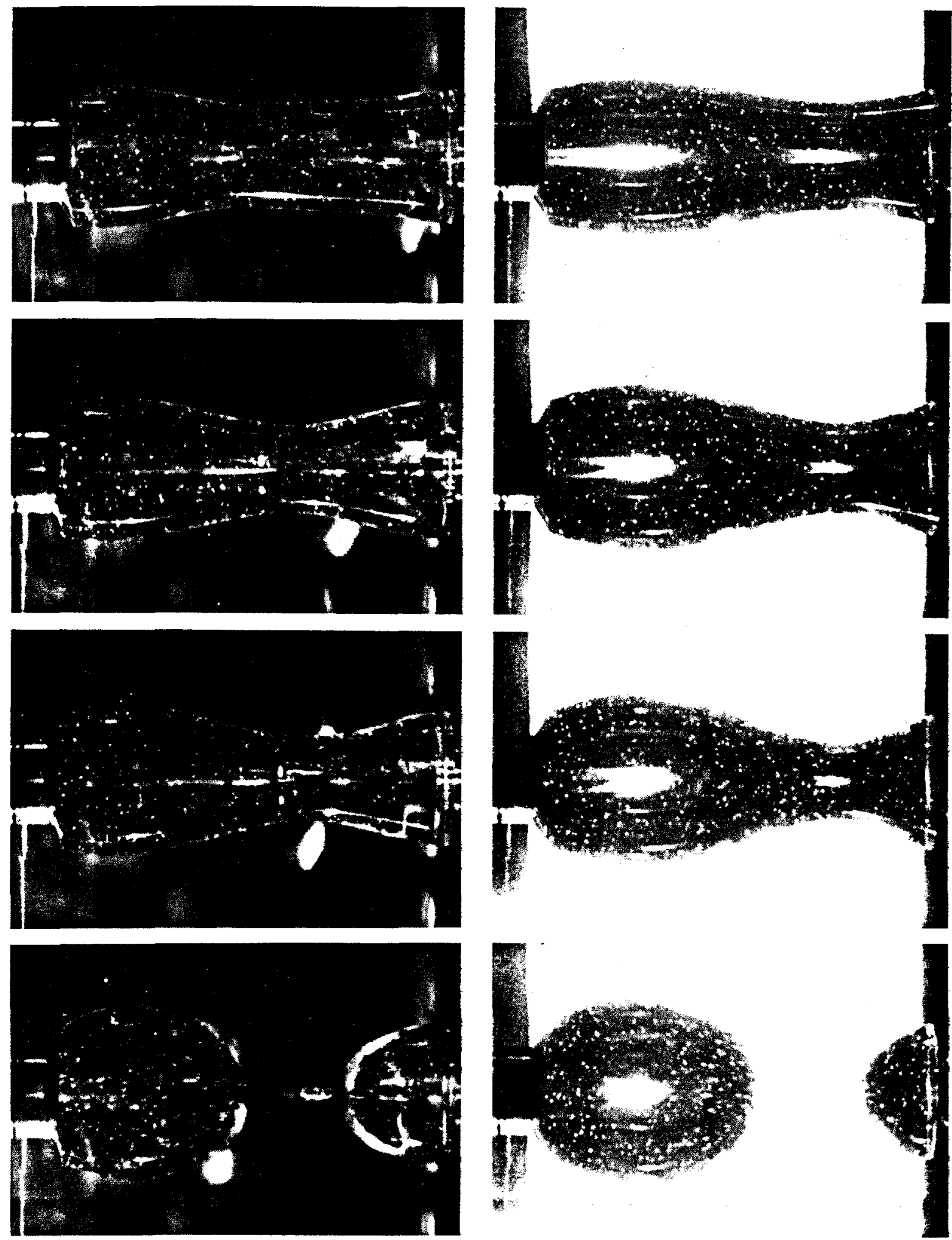

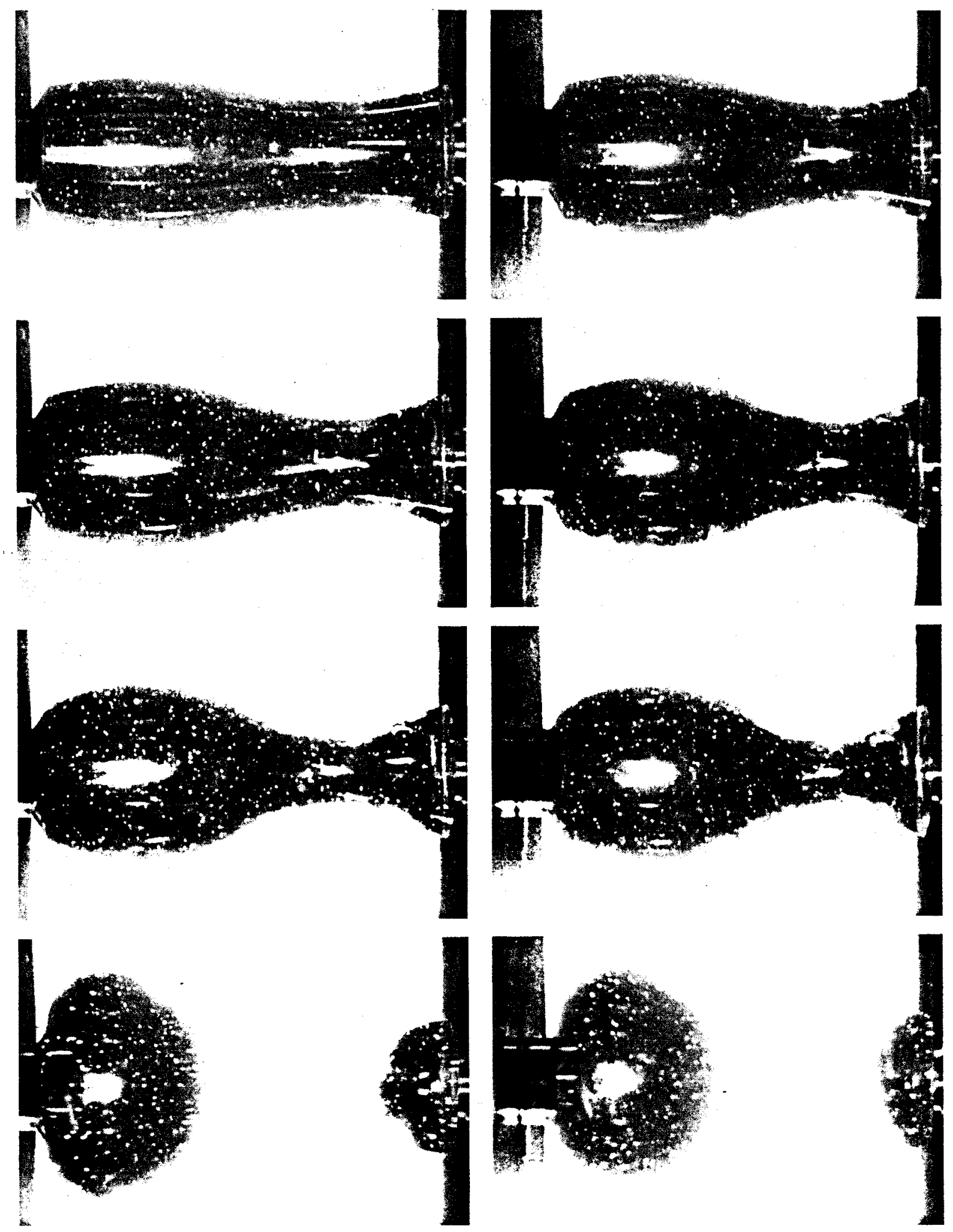

Fig. 2. Breaking sequences of long liquid bridges aboard Spacelab-D1. In each picture series times are, relative to the bottom picture. $-10 \mathrm{~s},-4 \mathrm{~s},-2 \mathrm{~s}$ and $0 \mathrm{~s} ; 1$ st column: Run $1 ; 2$ nd column: Run 3; 3rd column: Run 5; 4th column: Run 6. 
rately. Therefore, partial volumes obtained from the FLIZ experiment are compared with theoretical partial volumes obtained by solving a one-dimensional slice model for the dynamics of the liquid bridge. Since the ability of such slice model to predicts the dynamic behaviour of axisymmetric liquid bridges has been already tested through experiments in which microgravity was simulated by means of neutral buoyancy techniques $[4,5]$, one could expect similar agreement in the case of liquid bridges aboard a space station. The results presented here corroborate such assumption; experimental partial volumes obtained aboard Spacelab-D1 are in agreement with theoretical ones.

\section{Breaking sequences}

To analyze the different breaking sequences occurred during the FLIZ experiment, our sources of information have been a $16 \mathrm{~mm}$ film (shot at 0.5 frames per second) plus listings of the values of the experimental parameters (disk positions, rotation speeds, accelerometer measurements, etc.) and voice records of the communications between the spacecraft and the Earth.

Since the breaking of liquid bridges was not the main goal in FLIZ procedures, available information on these breakages is quite different from one to another breaking sequence. In addition, since liquid injection and removal was performed manually, the only available source of information concerning liquid bridge volumes are the pictures.

The status of the several breaking sequences is summarized in table 1. Observe that Run 1, Run 3 and Run 5 are reasonably well documented. On the other hand, the breaking in Run 4 cannot be analyzed because no pictures were shot after liquid column disruption. Concerning Run 2, the liquid bridge breaking happened just after vibration trials (an axial oscillatory motion $0.5 \mathrm{~mm}$ in amplitude and frequencies ranging from 0 to $1.6 \mathrm{~Hz}$ was imposed to the rear disk), and probably surprised the payload specialist (he stopped the film camera once vibration trials were finished, and there is a gap of about $1.5 \mathrm{~min}$ before the next picture). Finally, in the case of Run 6, an amount of data
Table 1

Availability of information concerning liquid bridge breakages during FLIZ experiment (Spacelab-D1)

\begin{tabular}{llrl}
\hline $\begin{array}{l}\text { Run } \\
\text { a) Pictures prior to } \\
\text { breaking available? shot after breaking available? }\end{array}$ & $\begin{array}{r}\text { Number of pictures Parameter listing } \\
\text { sul }\end{array}$ \\
\hline 1 & Yes & 10 & Yes \\
2 & No & 2 & Yes \\
3 & Yes & 15 & Yes \\
4 & Yes & 0 & Yes \\
5 & Yes & 22 & Yes \\
6 & Yes & 21 & No \\
\hline
\end{tabular}

a) Breaking sequences are numbered chronologically.

covering a period of about $8 \mathrm{~min}$, which includes a isorotation trial and the sequent breaking process, has been unfortunately lost; so, in this case, the only sources of information are the film plus the voice records.

To measure liquid bridge volumes, the film frames have been enlarged and the contours of liquid bridge interfaces digitized. Since the reference length is the disk diameter, and in some pictures disks are not very well defined (see fig. 2), this method of calculation of liquid bridge volumes becomes very sensitive to the accuracy in disk diameter measurements; therefore, when possible. several pictures showing the same liquid bridge configuration have been measured, the values of the volumes here reported are the average values.

In the following, unless otherwise stated. volumes are made dimensionless with $R_{0}^{3}$. Two additional parameters are introduced: the static Bond number $B=\rho g R_{0}^{2} / \sigma$, and the Weber number $W=\left(\rho \Omega^{2} R_{0}^{3} / \sigma\right)^{1 / 2}, g$ and $\Omega$ being the axial acceleration and the disk rotation speed, respectively.

To get an estimation of the value of Bond number during the FLIZ experiment (we consider only axial acceleration because lateral accelerations have higher order effects on stability limits [7]) the accelerometer readout supplied by DFVLR * has been used. The accelerometer gives the peak value sampled every second of accelera-

\footnotetext{
* Deutsche Forschungs- und Versuchsanstalt für Luft- und Raumfahrt e. V
} 
tion in a reference system having one of the axes parallel to the liquid column axis [8]. The peak values of axial acceleration $g^{+}$and $g^{-}$, respectively, during a period starting 3 min before breaking in Run 1, as well as the mean acceleration value $g_{\mathrm{m}}$, are shown in fig. 3 (although accelerometer data are available at each $1 \mathrm{~s}$, in this graph the interval between two sequent data is $5 \mathrm{~s}$, so that high frequency perturbations do not explicitly appear in fig. 3).

In the case of a linear system (as it is well known from random process theory [9]) the mean level of a stationary random perturbation is transmitted through the system as a constant signal; that is, the superposed random perturbation does not affect the relation MLI $/ \mathrm{MLO}=H(0)$ between the mean level input (MLI) and the mean level output (MLO), $H(\omega)$ being the transfer function which is characterized by the static response $H(0)$ (already known for almost cylindrical liquid bridges in a reasonable number of cases $[6,7,10-14])$ and the resonance frequencies $\omega[3,4]$. This principle can be used to describe the dynamics of a liquid bridge, which can be considered as a linear system provided the interface deforma-

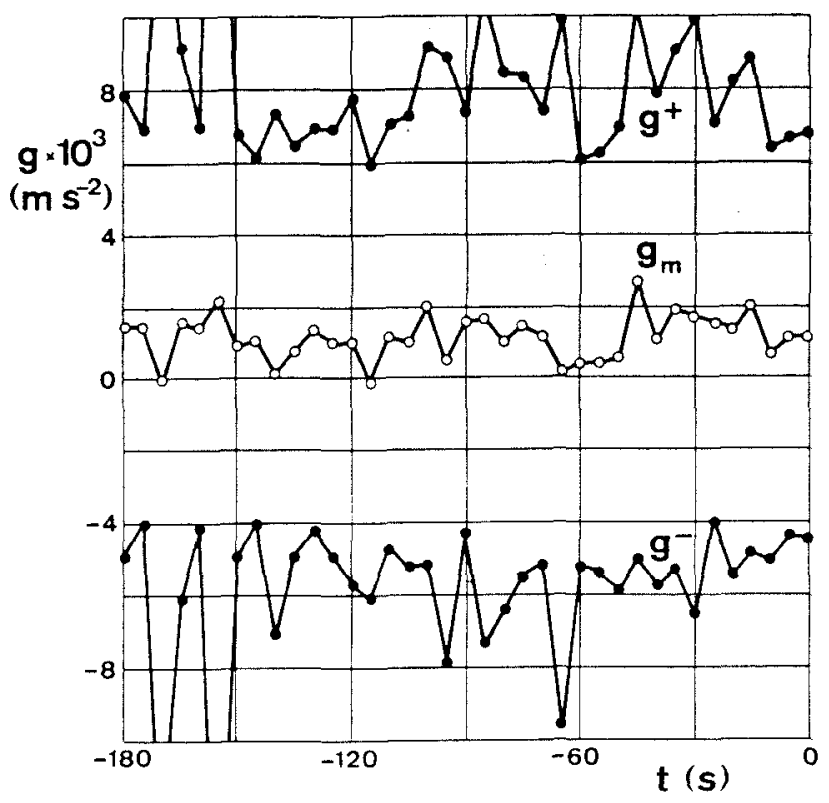

Fig. 3. Accelerometer data in the liquid bridge axial direction prior to the first liquid column breaking (Run 1). (๑) readout of accelerometer (peak values of acceleration sampled at $1 \mathrm{~s}$ interval. $g^{+}$and $g^{-}$, respectively); (O) mean acceleration $g_{m}$. tions are small enough. In this case, the input is the imposed perturbation and the output would be some measurement of the interface deformation.

Unfortunately, the previous considerations are not strictly applicable to the phenomenon under study because the liquid bridge breaking is a nonlinear process. However, the principal statement of the linear analysis (the main contribution to the output is the mean level of the input) can be used as a first insight in the problem, and to get an estimation of the mean level input a time averaged value, $g_{a}$, of the mean acceleration $g_{m}$ can be used.

The dependence of the averaging time of the average axial acceleration $g_{a}$, defined as

$g_{\mathrm{a}}(t)=\frac{1}{t_{\mathrm{b}}-t} \int_{t}^{t_{\mathrm{b}}} g_{\mathrm{m}}(\tau) \mathrm{d} \tau$,

is shown in fig. 4. In eq. (1), $t$ stands for the time and the subscript $b$ denotes the breaking instant (which coincides with the time origin, $t_{\mathrm{b}}=0$, in fig. 4). As it can be observed, this averaged value becomes nearly constant as the averaging time $t_{\mathrm{b}}-t$ increases. Therefore, an estimation of the value of Bond number during FLIZ experiment based in this averaged value $g_{\mathrm{a}}$ could be $B=$ $\rho g_{\mathrm{a}} R_{0}^{2} / \sigma \sim 10^{-2}$, this force being directed toward the feeding disk.

\subsection{Run I}

Basically, the beginning of the different trials of the FLIZ experiment was the formation of a long liquid column. To perform this task, FLIZ procedures stated that once a short liquid bridge was formed, the payload specialist should increase disk separation (by moving the feeding disk) until the desired slenderness $\Lambda$ is reached and, at the same time, inject working liquid into the liquid bridge to keep its volume nearly cylindrical $(V=2 \pi \Lambda)$ at any instant. This process is represented by line a in the stability diagram shown in fig. 5. The first breaking occurred because the injection law followed by the payload specialist (as already stated, injection was performed manually) was not that intended; therefore, the final configuration was very close to the minimum volume stability limit $[5,10]$ and thus, taking into account the value of 

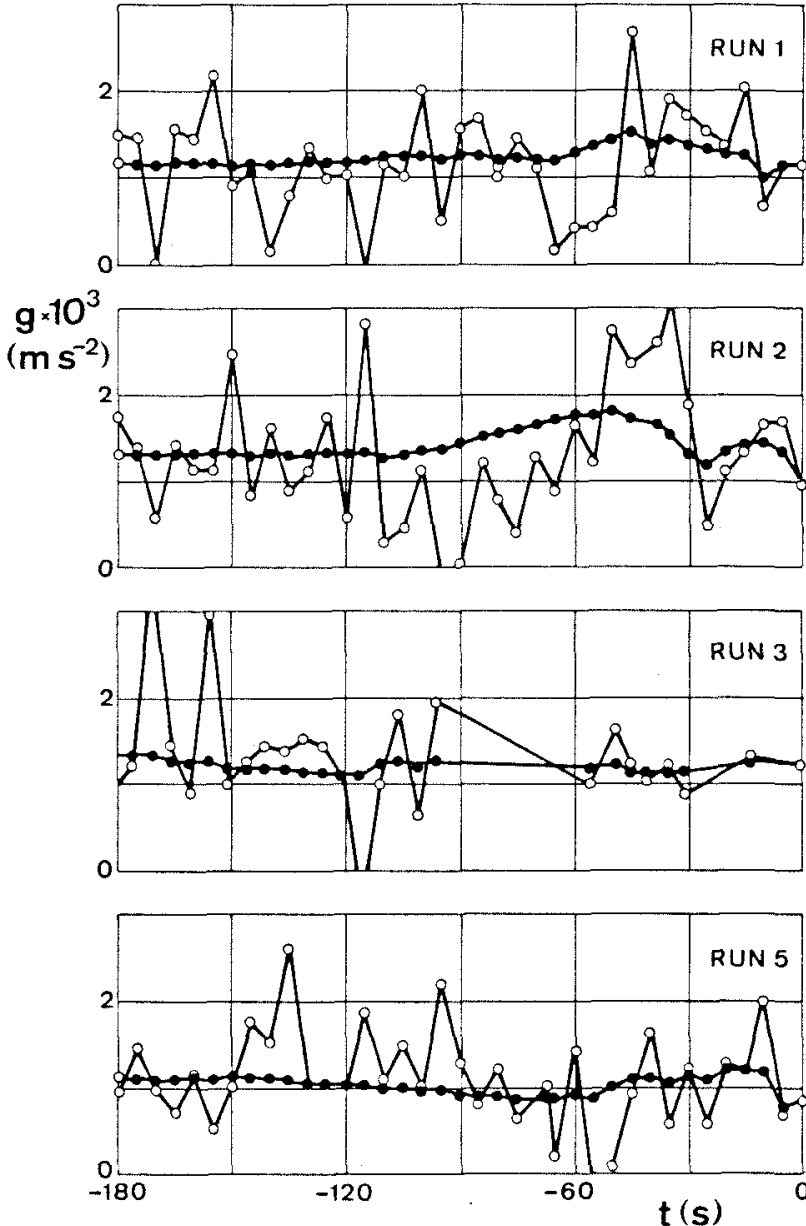

Fig. 4. Axial mean acceleration $g_{\mathrm{m}}(\mathrm{O})$ and averaged acceleration, $g_{\text {a }}(\bullet)$ prior to breakage for several liquid bridge sequences. In each plot the time origin corresponds to the breaking time with an error of $\pm 1 \mathrm{~s}$, except in the Run 2 case (see section 2.2).

the Bond number estimated in section 2. breaking in Run 1 happened because the liquid bridge became smaller than that of the minimum volume stability limit for the corresponding value of $B$.

The liquid bridge volume at the breaking configuration and the partial volume, both theoretical and as measured from photographs, are shown in table 2 (in all the cases the larger drop was the one anchored to the feeding disk, which is in agreement with the above mentioned sense of axial acceleration).

Theoretical partial volumes corresponding to experimental configurations have been obtained from fig. 6. which has been plotted from previous results already published in refs. [5,6]. Fig. 6 gives.

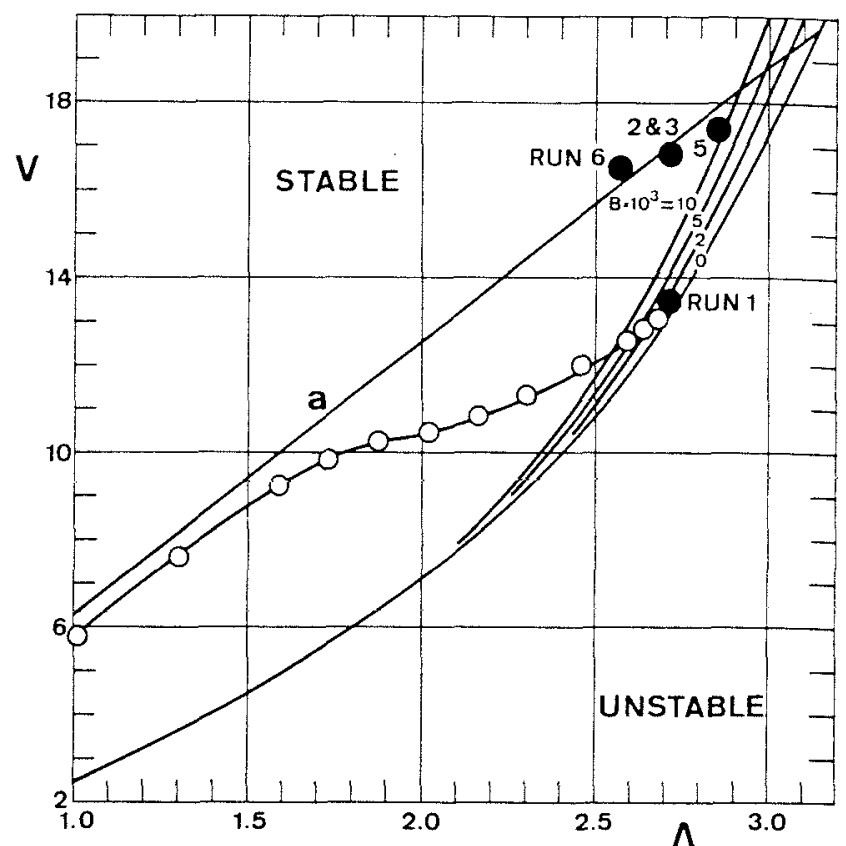

Fig. 5. Variation with Bond number $B$ of the minimum volume stability limit $V$. Line a corresponds to cylindrical volume liquid bridges, $V=2 \pi \Lambda$. Solid circles indicate where the liquid column breaking occurred; whereas open circles indicate the filling law manually followed in the first trial (Run 1).

for each value of the liquid bridge volume $V$ and the slenderness $\Lambda$, the partial volume $v_{\mathrm{p}}$, calculated by using a one-dimensional slice model for the dynamics of the liquid column [3-6]. Although $v_{\mathrm{p}}$ depends mainly on $V$ and $\Lambda$, in fig. 6 the dependence on the Bond number of the minimum volume stability limit is also represented (for each $B=$ constant curve the stable region is that placed above the curve).

Then, selecting the appropriated $\Lambda=$ constant

Table 2

Liquid bridge breakages during FLIZ experiment aboard Spacelab-D1

\begin{tabular}{lllll}
\hline Run & $A$ & $V \pm 0.3$ & $v_{\mathrm{pe}} \pm 0.004$ & $v_{\mathrm{pt}}$ \\
\hline 1 & 2.714 & 13.5 & 0.739 & $0.765 \pm 0.018$ \\
2 & 2.714 & 16.8 & 0.879 & $0.867 \pm 0.006$ \\
3 & 2.714 & 16.8 & 0.871 & $0.867 \pm 0.006$ \\
5 & 2.857 & 17.4 & 0.846 & $0.856 \pm 0.006$ \\
6 & 2.571 & 16.5 & 0.884 & $0.881 \pm 0.006$ \\
\hline
\end{tabular}

A: slenderness, $V$ : dimensionless liquid bridge volume. $l_{\text {pe }}$ : experimental partial volume. $v_{\mathrm{pt}}$ : theoretical partial volume. 


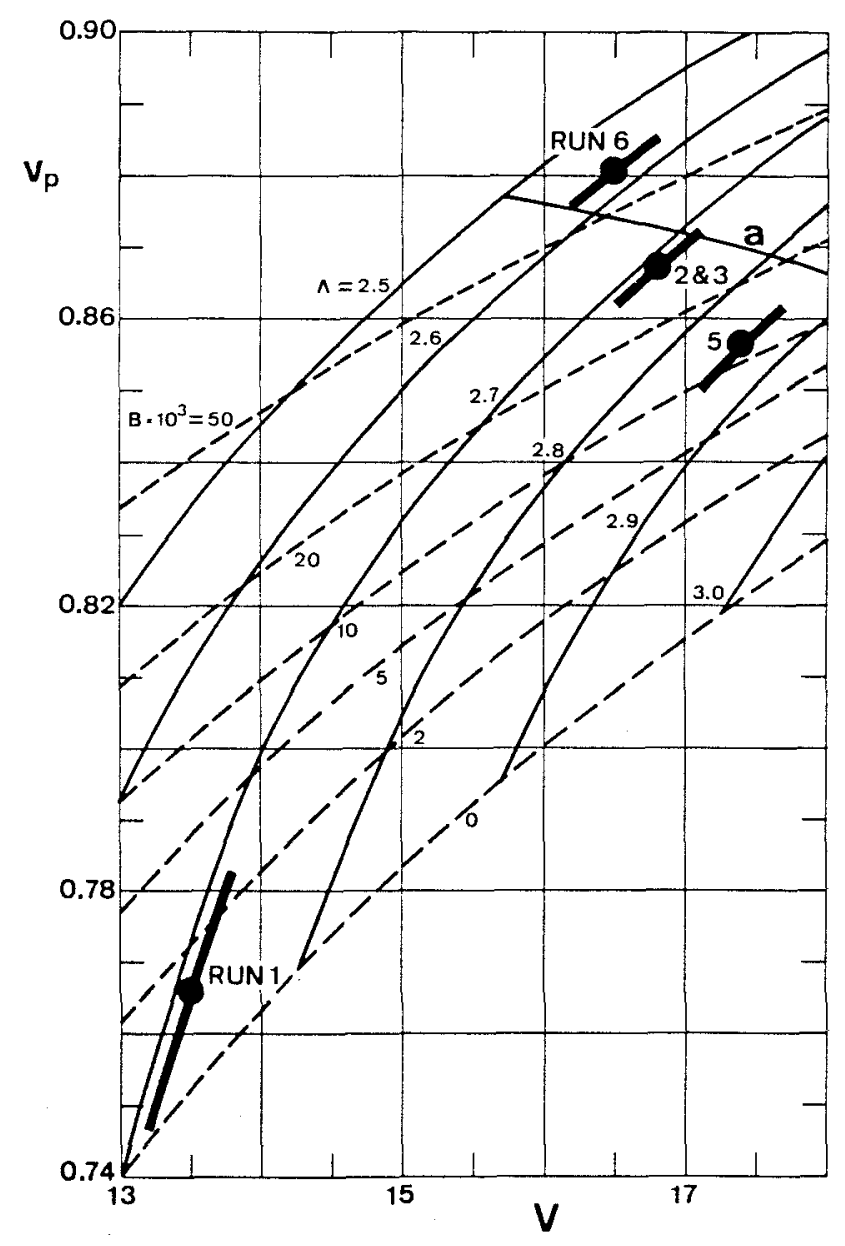

Fig. 6. Partial volume $v_{p}$ versus minimum volume stability limit $V$. Numbers on the curves indicate the values of the slenderness $\Lambda$ (-) and the Bond number $B$ ( $--(-)$. Line a corresponds to cylindrical volume liquid bridges. $V=$ $2 \pi \Lambda$. (•) Experimental liquid bridge configurations at breaking during the FLIZ experiment and the heavy lines indicate the accuracy in measurements of the liquid bridge volume.

curve and the liquid bridge volume $V$, obtained from measurements of pictures, the theoretical value of $v_{\mathrm{p}}$ results ${ }^{*}$. Observe that, since the slope of the $\Lambda$ curves increases as the liquid bridge volume decreases, the error in theoretical $v_{\mathrm{p}}$ values increases as $V$ decreases. In the first breaking (see table 2) measured values of $v_{\mathrm{p}}$ are about $3.5 \%$

\footnotetext{
* Note that. except in the case of Run 1, according to the mean value of Bond number estimated in section 2, all static configurations at breaking were stable. This indicates that axial mean acceleration was not the cause of these liquid bridge breakages, as will be explained later.
}

smaller than theoretical values (Run 1 gives the highest difference between experimental and theoretical values). The reason for this comparatively large difference could be that breaking in Run 1 happened after a liquid injection process (which tends to push the liquid towards the rear disk) combined with feeding disk separation (which has a similar effect) whereas theoretical results were obtained by assuming a liquid bridge at rest to which a perturbation (Bond number) is imposed at the initial time.

\subsection{Run 2}

As shown in table 1, Run 2 breaking is not very well documented. This breaking happened after oscillation trials, the resulting static liquid bridge configuration being stable (fig. 5). Liquid column breaking probably occurred because of an extremely high peak of axial acceleration. In any case, the main problem in the study of this breakage is that the breaking process was not filmed, so that it is not possible to correlate liquid bridge evolution with available acceleration recording. The values of axial mean acceleration, $g_{m}$, and time averaged acceleration, $g_{a}$, covering a period which roughly coincides with the not-filmed breaking process, are shown in fig. 4 ; in this plot the time origin has been fixed arbitrarily, coinciding with the first picture in which the liquid bridge appears broken in two drops.

Concerning partial volume, as shown in table 2 , the experimental value is about $1.4 \%$ higher than the theoretical value obtained from fig 6 .

\subsection{Runs 3, 5 and 6}

Each of these three bridge breakages took place after a isorotation trial. In these three cases, the static liquid bridge configurations were stable (figs. 5 and 6) but they become unstable when the prefixed rotation speeds were imposed (see fig. 7). Rotation speeds and slendernesses were selected in FLIZ procedures aiming to check the dependence on rotation speed of instability modes (axisymmetric mode or $\mathrm{C}$ mode [10-12]). Rotation speeds and slendernesses were $\Omega=10 \mathrm{rpm} . \Lambda=$ 2.857 (Run 5), $\Omega=13 \mathrm{rpm}, \Lambda=2.571$ (Run 6) 


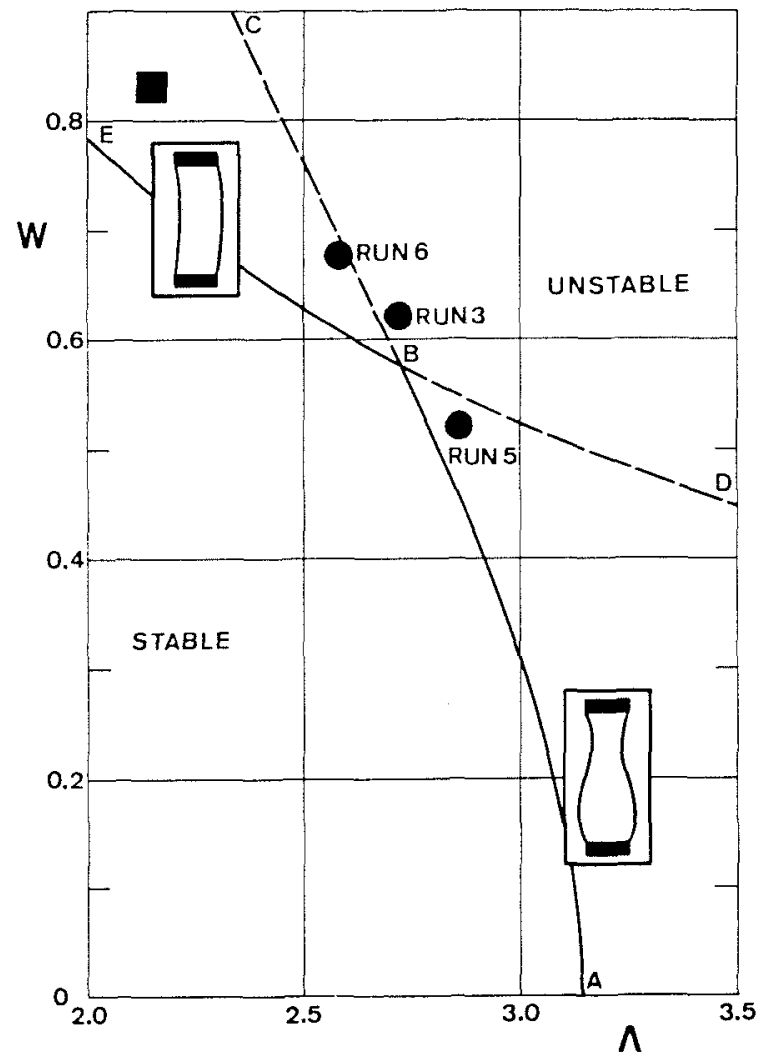

Fig. 7. Variation with Weber number $W$ of the maximum stable slenderness $A$ of cylindrical volume liquid bridges $(V=$ $2 \pi \Lambda)$ in gravitationless conditions $(B=0)$; line $A B C$ corresponds to axisymmetric stability limit, $\Lambda\left(1+W^{2}\right)^{1 / 2}=\pi$, whereas line DBE corresponds to non-axisymmetric ( $\mathrm{C}$ mode) stability limit, $2.1 W=\pi$ [12]. Therefore, there are four different regions in this stability diagram: the region below line $A B E$ is stable, but the region associated with the acute angle $A B D$ is a region in which there is instability to axisymmetric modes but stability with respect to $\mathrm{C}$ modes (the contrary occurs in the region $\mathrm{EBC}$ ); finally, in the region above line $\mathrm{DBC}$ both kind of instabilities take place. Solid circles indicate experimental liquid bridge configurations during the FLIZ experiment. The square corresponds to a fourth isorotation trial foreseen in FLIZ procedures which was not performed because of time constraints.

and $\Omega=12 \mathrm{rpm}, \Lambda=2.174$ (Run 3 ) which correspond to axisymmetric modes, to $\mathrm{C}$ modes and to the border between these two types of instability, respectively. A fourth isorotation trial, represented by a square in fig. 7 ( $\Omega=16 \mathrm{rpm}, A=2.143$ ) was foreseen in FLIZ procedures, but there was no time to carry it out during Spacelab-D1 Mission.

In these three breakages the breaking process was axisymmetric. This behaviour is obvious in the Run 5 case and even in the Run 3 case (fig. 7), but it is not so clear in the Run 6 breaking, where one could expect a $\mathrm{C}$ mode breaking. To explain this last case, additional perturbations, other than disk rotation, must be considered in the stability analysis. Fig. 8, which has been drawn after theoretical results reported in ref. [12], shows the maximum amplitude of the interface deformation $\epsilon$ of stable equilibrium shapes in the $\Lambda-W$ stability diagram. As it can be observed, except in the region bounded by lines $\mathrm{BE}$ and $\mathrm{BC}$, the perturbation level needed to excite $C$ mode instabilities is higher than that needed in the axisymmetric case. The main conclusion which can be obtained from fig. 8 is that any white noise, such as that existing during Spacelab-D1, acting on the liquid bridge $(\epsilon>0)$ moves point $\mathrm{B}$ effectively towards higher values of $W$, increasing the axisymmetric instability region and decreasing the $C$ mode instability one. Therefore, the axisymmetric behaviour in Run 6 breaking may be explained independently of the

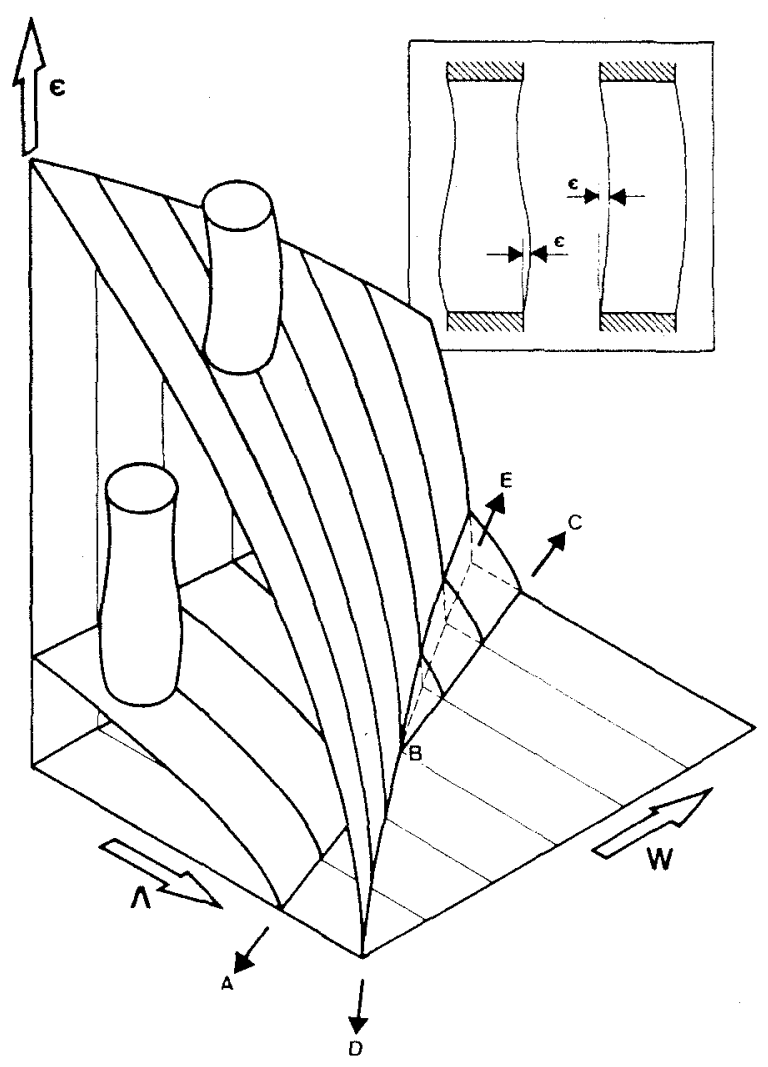

Fig. 8. Variation with the slenderness $\Lambda$ and the Weber number $W$ of the maximum interface deformation $\epsilon$ (defined as sketched in the insert) of stable equilibrium liquid bridges. 
value of the axial acceleration during Run 6, which would be assumed to be similar to that measured during the remaining liquid bridge breakages ( $B$ $\sim 10^{-2}$ ).

The evolution of the liquid bridge neck during the breaking processes under consideration is shown in fig. 9. Observe that in the case of Run 3 there is an oscillatory motion superposed to the breaking evolution. Concerning partial volumes (table 2) there is a surprising agreement between theoretical and experimental values (the maximum difference being $1.2 \%$ in the Run 5 case) in spite of the fact that in these three trials the liquid bridge was rotating whereas theoretical results were

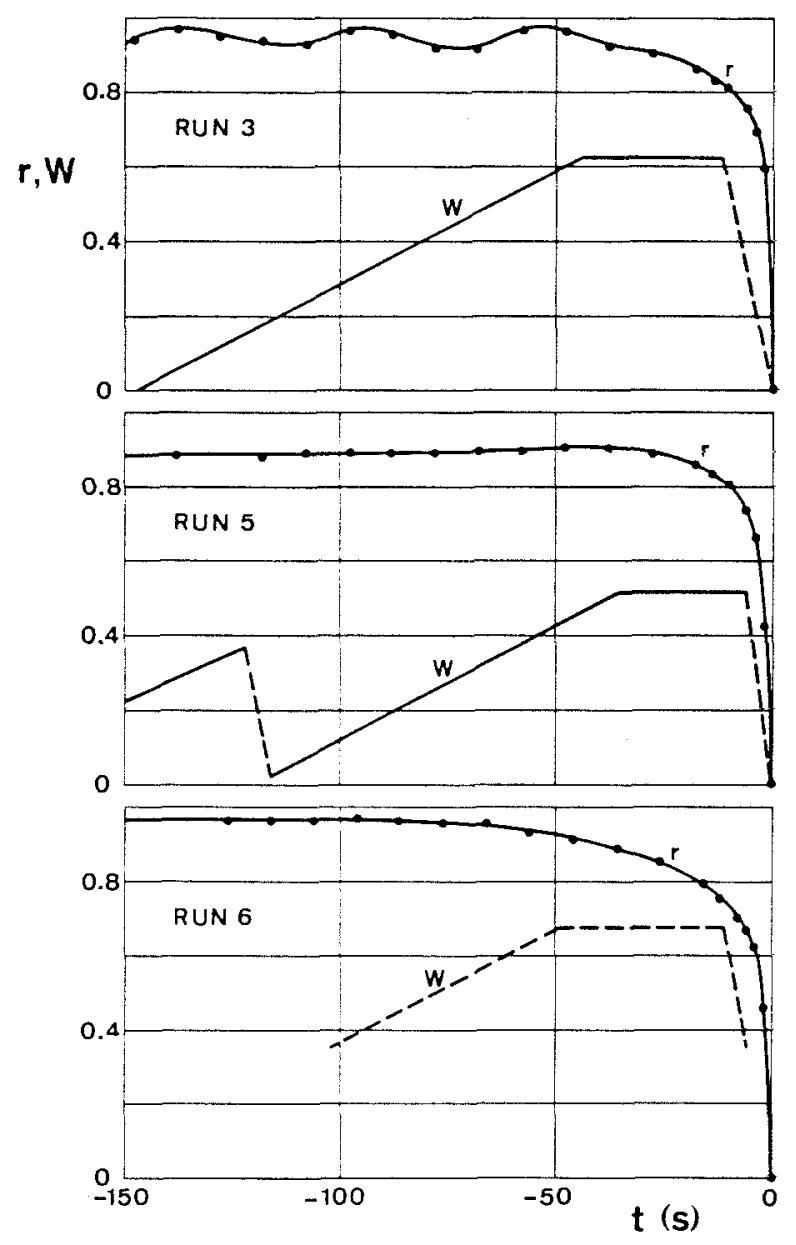

Fig. 9. Variation with time $t$ of the neck radius of the liquid bridge $r$ and the Weber number $W$ during isorotation trials performed aboard Spacelab-D1. Neck radii have been measured from pictures, whereas $W$ curves have been plotted after parameter listing; line type indicates when data were available $(\longrightarrow)$ or not $(--\infty)$ obtained by using a mathematical model which does not includes liquid column rotation. The reason for this agreement is that, once axisymmetric instabilities are excited, they govern the sequent liquid bridge evolution.

\section{Conclusions}

Five liquid bridge breaking sequences that occurred aboard Spacelab-D1 have been analyzed and compared with theoretical predictions. Although our attention has been focused mainly on breaking processes, the results obtained allow us to establish two main conclusions. First, theoretical stability studies predict accurately all of these liquid bridge breakages. Second, there is agreement between experimental and theoretical partial volumes, which seems to indicate that the breaking of a liquid bridge is a phenomenon mainly driven by surface tension (at least for the level of perturbations reached aboard Spacelab-D1), the different perturbations imposed to the liquid column, either accidental or intentionally, acting as a trigger that causes capillary instabilities to develop.

\section{Acknowledgement}

This work has been supported by the Spanish National Commission for Space Research. The authors acknowledge the collaboration of Dr. I. Martínez.

\section{References}

[1] P.R. Sahn and R. Jansen, Eds., Scientific Goals of the German Spacelab Mission D1 (DFVLR, Köln. 1985).

[2] A. Gonfalone, in: ESA SP-222 (ESA, Paris, 1984) p. 3.

[3] J. Meseguer. J. Fluid Mech. 130 (1983) 123.

[4] A. Sanz. J. Fluid Mech. 156 (1985) 101

[5] J. Meseguer and A. Sanz, J. Fluid Mech. 153 (1985) 83.

[6] J. Meseguer. J. Crystal Growth 73 (1985) 599.

[7] S.R. Coriell, S.C. Hardy and M.R. Cordes, J. Colloid Interface Sci. 60 (1977) 126.

[8] P. Zimmermann. The German Microgravity Laboratory for Material Science and Space Processing Experiments: Growth of Protein Crystals by Diffusion into Sodium 
Solution: A Material Science Experiment in Spacelab (MBB Space Division, Ottobrunn, Fed. Rep. of Germany, September 1979).

[9] J.S. Bendat and A.G. Piersol, Random Data: Analysis and Measurement Procedures (Wiley-Interscience, New York. 1971).

[10] I. Da Riva and I. Martínez, in: ESA SP-142 (ESA, Paris, 1979) p. 67.
[11] I. Martinez, in: COSPAR Space Research XVIII (Pergamon, Oxford, 1978) p. 519.

[12] J.M. Vega and J.M. Perales, in: ESA SP-191 (ESA, Paris, 1983) p. 247.

[13] J. Meseguer, in: ESA SP-222 (ESA, Paris, 1984) p. 297.

[14] J. Meseguer, L.A. Mayo, J.C. Llorente and A. Fernández, J. Crystal Growth 73 (1985) 609. 